\title{
Genetic testing for aortic valve stenosis
}

\author{
Yeltay Rakhmanov ${ }^{1}$, Paolo Enrico Maltese ${ }^{1 \star}$, Alessandra Zulian ${ }^{2}$, Stefano Paolacci ${ }^{2}$, Tommaso Beccari ${ }^{3}$, \\ Munis Dundar ${ }^{4}$ and Matteo Bertelli ${ }^{1,2}$
}

\begin{abstract}
Aortic valve stenosis (AVS) is a congenital aortic defect in which the aortic lumen narrows due to thickening or calcification of the aortic valve without obstructing left ventricular outflow. Depending on the site of obstruction, AVS is classified as valvular, sub-valvular or supra-valvular. The prevalence of AVS is about $3 \%$ and increases with age. One in eight persons over the age of 75 years has moderate or severe AVS. AVS has autosomal dominant inheritance. It can be associated with mutations in the following genes: NOTCH1, SMAD6, SMAD4, and ELN. This Utility Gene Test was developed on the basis of the analysis of the literature and existing diagnostic protocols. It is useful for confirming diagnosis, as well as for differential diagnosis, couple risk assessment and access to clinical trials, when available.
\end{abstract}

Keywords: Aortic valve stenosis, NOTCH1, SMAD6, ELN, SMAD4, EBTNA UTILITY GENE TEST

'MAGI's Lab, Rovereto, Italy

${ }^{2}$ MAGI Euregio, Bolzano, Italy

${ }^{3}$ Department of Pharmaceutical Sciences, University of Perugia, Perugia, Italy

${ }^{4}$ Department of Medical Genetics, Erciyes University Medical School, Kayseri, Turkey

*Corresponding author: P. E. Maltese E-mail: paolo.maltese@assomagi.org

DOI: 10.2478/ebtj-2018-0040
C 2018 Authors. This work was licensed under the Creative Commons AttributionNonCommercial-NoDerivs 3.0 License.

\section{Aortic valve stenosis}

(other synonyms: valvular aortic stenosis, aortic valve disease)

\section{General information about the disease}

Aortic valve stenosis (AVS) is a congenital aortic defect characterised by considerable narrowing of aortic lumen due to thickening or calcification of the aortic valve without obstruction to left ventricular outflow. Depending on the site of obstruction, AVS is classified as valvular, sub-valvular, or supra-valvular (1). Valvular aortic stenosis generally occurs as a consequence of valves with two leaflets rather than three, known as bicuspid aortic valve (BAV) and is the most common cardiovascular malformation (2). There is a section on BAV in this special issue. Sub-valvular aortic stenosis presents as a membranous or fibromuscular ring below the aortic valve, either isolated or associated with other congenital anomalies $(3,4)$. Supra-valvular aortic stenosis is a narrowing of the ascending aorta in the form of an hourglass deformity or diffuse hypoplasia at the margin of the Valsalva sinuses, just above the junction of coronary arteries (5-7).

Epidemiological studies have shown that more than one in eight people aged 75 and older have moderate or severe AVS. The prevalence of moderate or severe AVS is $3 \%$ and increases with age (8).

The diagnosis of AVS is established in the first year of life. Patients may have the following symptoms: dyspnoea, chest pain, myocardial infarction, stroke or even sudden death during exercise. Due to elastin deficiency, patients may have hoarse voice, hernias, lax skin, the signs of premature ageing, stiffness of joints, and even vascular hypertension (7).

Diagnostic work-up includes clinical assessment to identify symptoms, echocardiogram, electrocardiogram, chest radiogram, integration of septal and color Doppler with two- 
dimensional echocardiography, angiography by retrograde femoral arterial catheterization and angio-NMR (nuclear magnetic resonance), spin-echo MR imaging, CT, diagnostic catheterization and genetic testing.

Differential diagnosis should consider aortic coarctation, interruption of the aortic arch and hypoplastic left heart syndrome.

AVS has autosomal dominant inheritance with incomplete penetrance and variable expressivity.

The etiology of AVS is linked to dysregulation of NOTCH and TGF-beta molecular pathways that play a fundamental role in aortic valve genesis (9). Mutant genes in syndromic and isolated AVS, such as NOTCH1, SMAD6 and SMAD4, are involved in these pathways, whereas truncating mutations in ELN cause supravalvar aortic stenosis through reduced elastin production and subendothelial proliferation of smooth muscle, contributing to obstructive arterial disease (10).

\section{Autosomal dominant non-syndromic AVS}

- Aortic valve disease 1 (AVD1, OMIM disease 109730) NOTCH1 (OMIM gene 190198);

- Aortic valve disease 2 (AVD2, OMIM disease 614823) SMAD6 (OMIM gene 602931);

- Supravalvar aortic stenosis (SVAS, OMIM disease 185500) ELN (OMIM gene 130160).

\section{Autosomal dominant syndromic AVS}

- Myhre syndrome (MYHRS, OMIM disease 139210) SMAD4 (OMIM gene 600993).

Pathogenic variants be include missense, nonsense, splicing, small insertions, deletions or indels.

\section{Aims of the test}

- To determine the gene defect responsible for the disease;

- To confirm clinical diagnosis;

- To assess the recurrence risk and perform genetic counselling for at-risk/affected individuals.

\section{Test characteristics}

\section{Specialist centers/ Published Guidelines}

The test is listed in the Orphanet database and is offered by 2 accredited medical genetic laboratories in the EU, and in the GTR database, offered by 1 accredited medical genetic laboratories in the US.

Guidelines for clinical use of the test are described in Genetics Home Reference (ghr.nlm.nih.gov).

\section{Test strategy}

A multi-gene next generation sequencing panel is used for the detection of nucleotide variations in coding exons and flanking introns of the above genes.

Potentially causative variants and regions with low coverage are Sanger-sequenced. Sanger sequencing is also used for family segregation studies.
To perform molecular diagnosis, a single sample of biological material is normally sufficient. This may be $1 \mathrm{ml}$ peripheral blood in a sterile tube with $0.5 \mathrm{ml} \mathrm{K}$ EDTA or $1 \mathrm{ml}$ saliva in a sterile tube with $0.5 \mathrm{ml}$ ethanol $95 \%$. Sampling rarely has to be repeated.

Gene-disease associations and the interpretation of genetic variants are rapidly developing fields. It is therefore possible that the genes mentioned in this note may change as new scientific data is acquired. It is also possible that genetic variants today defined as of "unknown or uncertain significance" may acquire clinical importance.

\section{Genetic test results \\ Positive}

Identification of pathogenic variants in the above genes confirms the clinical diagnosis and is an indication for family studies.

A pathogenic variant is known to be causative for a given genetic disorder based on previous reports, or predicted to be causative based on loss of protein function or expected significant damage to proteins or protein/protein interactions. In this way it is possible to obtain a molecular diagnosis in new/other subjects, establish the risk of recurrence in family members and plan preventive and/or therapeutic measures.

\section{Inconclusive}

Detection of a variant of unknown or uncertain significance (VUS): a new variation without any evident pathogenic significance or a known variation with insufficient evidence (or with conflicting evidence) to indicate it is likely benign or likely pathogenic for a given genetic disorder. In these cases, it is advisable to extend testing to the patient's relatives to assess variant segregation and clarify its contribution. In some cases, it could be necessary to perform further examinations/tests or to do a clinical reassessment of pathological signs.

\section{Negative}

The absence of variations in the genomic regions investigated does not exclude a clinical diagnosis but suggests the possibility of:

- alterations that cannot be identified by sequencing, such as large rearrangements that cause loss (deletion) or gain (duplication) of extended gene fragments;

- sequence variations in gene regions not investigated by this test, such as regulatory regions (5' and $3^{\prime}$ UTR) and deep intronic regions;

- variations in other genes not investigated by the present test.

\section{Unexpected}

Unexpected results may emerge from the test, for example information regarding consanguinity, absence of family correlation or other genetically-based diseases.

\section{Risk for progeny}

In autosomal dominant transmission, the probability that an affected carrier transmit the variant to his/her children is $50 \%$ in any pregnancy, irrespective of the sex of the child conceived. 
Incomplete penetrance and/or variable expressivity may influence the manifestation of clinical symptoms in individuals harboring a disease-causing variation.

\section{Limits of the test}

The test is limited by current scientific knowledge regarding the gene and disease.

Analytical sensitivity (proportion of positive tests when the genotype is truly present) and specificity (proportion of negative tests when the genotype is not present)

NGS: Analytical sensitivity $>99.99 \%$, with a minimum coverage of 10X; Analytical specificity $99.99 \%$.

SANGER: Analytical sensitivity $>99.99 \%$; Analytical specificity 99.99\%.

Clinical sensitivity (proportion of positive tests if the disease is present) and clinical specificity (proportion of negative tests if the disease is not present)

Clinical sensitivity: the variations in the aforementioned genes are linked to AVS, but may be individual variations (identified in one or few families) and total epidemiological data is therefore not available.

Clinical sensitivity will be estimated based on internal cases.

Clinical specificity is estimated at approximately $99 \%$ (11).

\section{Prescription appropriateness}

The genetic test is appropriate when:

a) the patient meets the diagnostic criteria for AVS;

b) the sensitivity of the test is greater than or equal to that of tests described in the literature.

\section{Clinical utility}

Clinical management

Confirmation of clinical diagnosis

Differential diagnosis Yes

Couple risk assessment Yes

Availability of clinical trials can be checked on-line at https://clinicaltrials.gov/

\section{References}

1. Czarny MJ, Resar JR. Diagnosis and Management of Valvular Aortic Stenosis. Clinical Medicine Insights Cardiology 2014; 8(Suppl 1): $15-24$.

2. Roberts WC. The congenitally bicuspid aortic valve. A study of 85 autopsy cases. Am J Cardiol 1970; 26(1): 72-83.

3. Kleinert S, Ott DA, Geva T. Critical discrete subaortic stenosis in the newborn period. Am Heart J1993; 125(4): 1187-89.

4. Darcin OT, Yagdi T, Atay Y, Engin C, Levent E, Buket S, Alayunt EA. Discrete Subaortic Stenosis: Surgical Outcomes and Follow-Up Results. Texas Heart Institute Journal 2003; 30(4): 286-92.

5. Vaideeswar P, Shankar V, Deshpande JR, Sivaraman A, Jain N. Pathology of the diffuse variant of supravalvar aortic stenosis. Cardiovasc Pathol 2001; 10(1): 33-37.

6. Stamm C, Friehs I, Ho SY, Moran AM, Jonas RA, del Nido PJ. Congenital supravalvar aortic stenosis: a simple lesion? Eur J Cardiothorac Surg 2001; 19(2): 195-02.

7. Patrice B. Supravalvular aortic stenosis. Orphanet encyclopedia 2003; https://www.orpha.net/data/patho/GB/uk-SVAS.pdf.

8. Nkomo VT, Gardin JM, Skelton TN, Gottdiener JS, Scott CG, Enriquez-Sarano $M$. Burden of valvular heart diseases: a population-based study. Lancet 2006; 368(9540): 1005-11.

9. Dutta $\mathrm{P}$, Lincoln J. Calcific aortic valve disease: a developmental biology perspective. Current Cardiology Reports 2018; 20(4): 21.

10. Li DY, Brooke B, Davis EC, Mecham RP, Sorensen LK, Boak $B B$, Eichwald E, Keating MT. Elastin is an essential determinant of arterial morphogenesis. Nature 1998; 393(6682): 276-80.

11. Chen B, Gagnon M, Shahangian S, Anderson NL, Howerton DA, Boone JD; Centers for Disease Control and Prevention (CDC). Good Laboratory Practices for Molecular Genetic Testing for Heritable Diseases and Conditions. MMWR Recomm Rep 2009; 58(RR6): 1-37. 